Tecno Lógicas

ISSN 0123-7799

Vol. 18, No. 35, pp. 83-91

Julio-diciembre de 2015

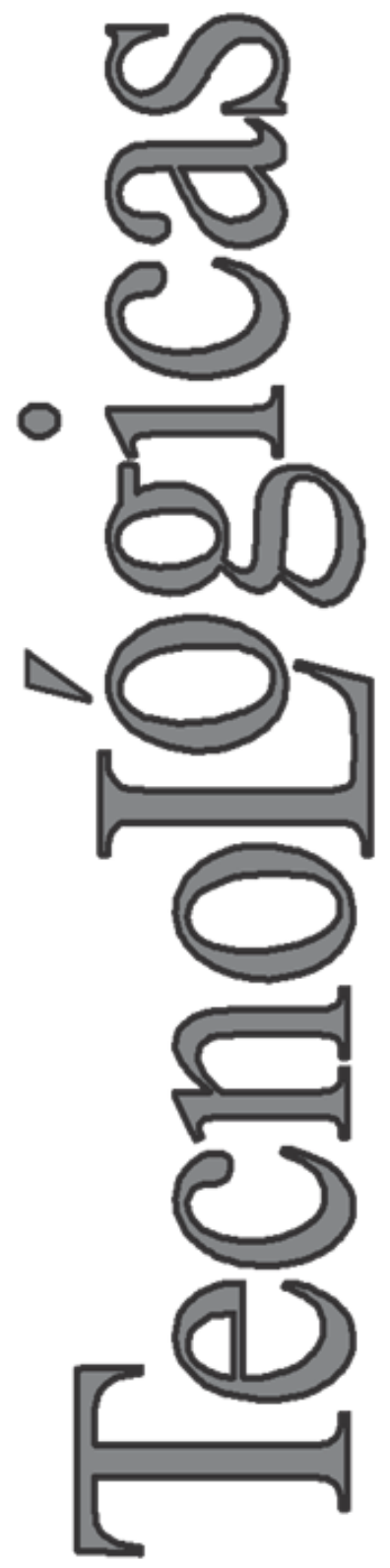

(C) Copyright 2015 por autores y Tecno Lógicas Este trabajo está licenciado bajo una Licencia Internacional Creative Commons Atribución (CC BY)

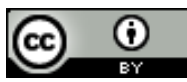

\section{Elaboración de recubrimientos base cobre sobre un sustrato de porcelana mediante proyección térmica con Ilama oxiacetilénica}

\section{Copper alloy coatings manufactured by oxy- fuel thermal spray onto a porcelain substrate}

\author{
Daniela Soto-Martínez ${ }^{1}$, Carolina Parra-Velásquez², \\ María E. López-Gómez ${ }^{3}$, Carlos F. Vásquez-Jiménez ${ }^{4}$ \\ y Fabio Vargas-Galvis ${ }^{5}$
}

Recibido: 26 de febrero de 2015,

Aceptado: 12 de junio de 2015

Cómo citar / How to cite

D. Soto-Martínez et al., "Elaboración de recubrimientos base cobre sobre un sustrato de porcelana, mediante proyección térmica con llama oxiacetilénica”, Tecno Lógicas, vol. 18, no. 35, pp. 83-91, 2015.

1 Estudiante Ingeniería de Materiales, Grupo GIMACYR, Universidad de Antioquia, Medellín-Colombia, danisomat@hotmail.com

2 Estudiante Ingeniería de Materiales, Grupo GIMACYR, Universidad de Antioquia, Medellín-Colombia, carolina.parrav@udea.edu.co

3 Ph. D. en Ciencia y Tecnología de Materiales, Grupo GIMACYR, Universidad de Antioquia, Medellín-Colombia, esperanza.lopez@udea.edu.co

4 Máster en Ingeniería de Materiales, Electroporcelana Gamma, Organización Corona, Medellín-Colombia, cfvasquez@corona.com.co

5 Ph. D. en Ingeniería y en Materiales Cerámicos y Tratamiento de Superficies, Grupo GIMACYR, Universidad de Antioquia, MedellínColombia, fabio.vargas@udea.edu.co 


\title{
Resumen
}

Se desarrolló un sistema constituido por un sustrato cerámico de porcelana y un recubrimiento de una aleación de cobre, con el fin de proporcionar una superficie conductora de la electricidad en partes específicas del sustrato. Para ello se analizó previamente la mejor manera de preparar la superficie del sustrato para que las partículas de la aleación de cobre se adhieran a él. Para la elaboración del recubrimiento se utilizó un polvo marca Eutectic Castolin ${ }^{\mathrm{TM}}$ referencia 21071, el cual fue proyectado utilizando llamas oxiacetilénicas producidas a partir de dos diferentes relaciones volumétricas de combustible-comburente $(1: 1,15$ y 1:2,5), dando como resultado una llama reductora y una oxidante respectivamente. El efecto de la preparación superficial del sustrato, así como el del espesor del recubrimiento y el de la energía de la llama sobre la estructura y adherencia del recubrimiento fue evaluado, encontrando que los recubrimientos obtenidos utilizando una llama reductora tienen baja adherencia al sustrato cerámico preparado superficialmente, y un alto nivel de porosidad en su estructura, mientras que aquellos elaborados utilizando una llama oxidante tienen la mayor adherencia y su estructura es homogénea (baja porosidad). Finalmente, los recubrimientos depositados con los dos tipos de llama sobre sustratos sin preparación superficial presentaron muy baja o nula adherencia.

\section{Palabras clave}

Proyección térmica, adherencia, recubrimientos base cobre, sustrato cerámico.

\begin{abstract}
A coated system consisting of a ceramic substrate and a coating of a copper alloy was developed in order to provide electrically conductive properties to specific surface of the substrate. The coatings were elaborated by oxy-fuel thermal spraying processes. The best way to prepare the surface of the substrate was previously determined to achieve a sufficient adherence of copper alloy particles on the ceramic substrate. In order to manufacture the coatings an Eutectic Castolin ${ }^{\mathrm{TM}} 21071$ powder was used, the oxy-fuel flames were produced from two different volumetric acetylene-oxygen ratios (1:1.15 and 1:2.5) obtaining a reducing and oxidizing flame respectively. The effect of surface preparation of the substrate, the coating thickness and the energy of the flame on the structure and coating adhesion were evaluated. It was found that the coatings obtained using a reducing flame presented lower adhesion values to the ceramic substrate and a higher level of porosity in its structure, while those made using an oxidizing flame presented greater adhesion and an homogenous structure (low porosity). Finally, a very low adhesion values were obtained on the non-prepared substrates with both flames.
\end{abstract}

\section{Keywords}

Thermal spray, adhesion, ceramic substrate, surface treatment, cooper-based coatings. 


\section{INTRODUCCIÓN}

En términos generales, se puede definir a los aislantes eléctricos como materiales que reducen el paso de electricidad de un punto a otro, estos comúnmente están fabricados con materiales que no conducen la electricidad, como la silicona, el vidrio y las electroporcelanas [1]. Los aisladores cerámicos o de electroporcelana tienen aplicación en las líneas de baja y alta tensión y son de gran importancia para la industria de la distribución y la transmisión eléctrica debido a su resistencia dieléctrica, mecánica y química. Estas propiedades están directamente relacionadas con su procesamiento y con las características de las materias primas que se utilizan para su fabricación, como el feldespato, el caolín, el cuarzo y las arcillas [1].

A pesar del amplio desarrollo que han tenido los aisladores eléctricos y su amplio uso en las líneas de distribución y transmisión, las descargas localizadas que pueden conducir a un efecto corona y a problemas de radio interferencia continúan siendo un problema; por lo tanto, el objetivo de este trabajo fue proporcionar características conductoras en zonas específicas de ciertos materiales cerámicos, aplicando una capa de una aleación de cobre utilizando la técnica de proyección térmica con llama oxiacetilénica [2]. El cobre es un material conductor de electricidad por excelencia y sus aleaciones (especialmente de cobrealuminio) son ampliamente utilizadas en proyección térmica para la elaboración de recubrimientos con buen desempeño en condiciones de desgaste por fricción y como medio de protección de la corrosión, en piezas como asientos de rodamientos, sellos de bombas, ejes de motores, ejes de propulsores de barcos y reconstrucción de piezas de cobre desgastadas [3].

Por su parte, la proyección térmica es quizás uno de los procesos más utilizados para la elaboración de recubrimientos con buenas características estructurales sobre piezas de diversos tamaños y geometrías a un bajo costo, sin embargo, la calidad de la capa depositada depende de una buena selección de las materias primas y de los parámetros utilizados para la proyección, ya que de esto depende el grado de fusión que experimenten las partículas que conforman la estructura del recubrimiento, así como su empaquetamiento, cohesión entre capas y adhesión al sustrato [4]. Dentro de los parámetros involucrados en el proceso de elaboración de recubrimientos, los más relevantes para obtener buenos resultados son: la preparación superficial del sustrato, el flujo del polvo utilizado para elaborar el recubrimiento, y la entalpía de la llama utilizada para fundir el polvo de partida [4].

La preparación superficial del sustrato es requerida para obtener un buen anclaje mecánico de las partículas proyectadas sobre el sustrato y por lo tanto es fundamental para la adherencia del recubrimiento. Generalmente la preparación superficial del sustrato se describe a través de la rugosidad media $(\mathrm{Ra})$, la cual representa la media de las irregularidades de la superficie [4], [5].

Por su parte, el flujo y la continuidad de la alimentación de polvo de partida se ve afectada por sus características físicas (morfología y distribución del tamaño de partícula); estos idealmente, deberían tener una distribución granulométrica adecuada y estar libres de partículas extremadamente finas (submicrométricas o nanométricas), deben tener el menor contenido de humedad posible y su morfología debería ser esférica ya que partículas de forma irregular no fluyen tan fácilmente por los conductos de las antorchas de proyección térmica debido a que se empaquetan más fácilmente entre ellas [6]. Una baja fluidez de las partículas puede generar problemas de continuidad en la capa de recubrimiento, lo cual en el caso de recubrimientos conductores podría generar variaciones importantes en la resistencia eléctrica [7].

Por otro lado, en el proceso de proyección térmica por combustión, se utiliza la 
energía química de gases combustibles y comburentes para la generación de la llama encargada de fundir las partículas proyectadas. Las antorchas oxiacetilénicas utilizan la mezcla de acetileno $\left(\mathrm{C}_{2} \mathrm{H}_{2}\right)$ y oxígeno $\left(\mathrm{O}_{2}\right)$, que en una relación estequiométrica se obtiene una llama que alcanza una temperatura de hasta $3113^{\circ} \mathrm{C}$ a 1 atm de presión [4]. Una llama oxidante o altamente oxidante es en la que se aplica un exceso de comburente y como resultado se obtiene una zona de reacción primaria (cono de la llama) mucho más larga que en una llama reductora o en una estequiométrica, por lo que las partículas tienen un mayor tiempo de residencia en la zona más caliente de la llama y por lo tanto, pueden lograr un mejor calentamiento.

Es de anotar que tanto en las llamas con exceso de comburente (oxidantes), como en aquellas con exceso de combustible (reductoras), dicho exceso no produce calor de reacción y por el contrario consume parte del calor generado por la reacción, dando lugar a una disminución en la temperatura de la zona primaria de reacción que es la zona más caliente de la llama. De acuerdo con lo anterior, una llama reductora con una relación volumétrica de acetileno:oxígeno de 1:1,15 alcanzará una temperatura máxima en la zona de reacción primaria de aproximadamente $3080^{\circ} \mathrm{C}$, mientras que la llama oxidante alcanza una temperatura máxima en la misma zona de aproximadamente $3070^{\circ} \mathrm{C}$. En las llamas reductoras, además de la menor temperatura en la zona de reacción primaria, la longitud de dicha zona es más corta que la de las llamas oxidantes [4], [8].

La temperatura de la llama influye en los esfuerzos residuales que se generan en el sustrato durante el proceso de proyección, dado que los metales y los cerámicos a menudo difieren en gran medida en los coeficientes de expansión térmica, las tensiones mecánicas resultantes en la interfaz metal-cerámico son de una magnitud considerable [9]. En los sistemas de recubrimientos metal/cerámico, la estructura $\mathrm{y}$ composición de la interfaz juega un papel importante en las propiedades del material obtenido, pues esta es la que debe soportar los esfuerzos mecánicos o eléctricos sin fallar, es por esto que, una buena estructura e interacción de sus componentes es crucial para un buen desempeño del material. Debido al alto potencial de aplicación de estos sistemas, ha venido creciendo su estudio y desarrollo en amplias aplicaciones (sistemas de empaques electrónicos para el procesamiento de información, tecnología de películas delgadas, entre otras) [10].

Rühle [10] utilizó varias técnicas para analizar la interfaz metal/cerámico y sus interacciones en sistemas $\quad \mathrm{Nb}^{-\mathrm{Al}_{2} \mathrm{O}_{3}}$ y $\mathrm{Cu} / \mathrm{Al}_{2} \mathrm{O}_{3}$, encontrando que los esfuerzos residuales en el primer sistema son despreciables debido a que los coeficientes de dilatación térmica de los materiales son casi iguales; mientas que en el segundo sistema se obtiene una muy pobre adherencia. En estos sistemas metal/cerámico, aparte de obtener pobres resultados de adherencia se puede dar fractura de los sustratos; Kuneman et al. [11], patentaron un método para depositar recubrimientos de cobre sobre sustratos cerámicos de alúmina, sin embargo, tuvieron problemas de agrietamiento de los sustratos durante y después del enfriamiento del sistema (recubrimiento-sustrato). Esto se debe a que el cobre, que tiene un mayor coeficiente de expansión térmica que la alúmina, es sometido a un estiramiento sustancial o esfuerzos de tracción, mientras que en la parte superior del sustrato de alúmina, se generan esfuerzos de compresión [11]. Por su parte, Kinsho et al., plantearon que es posible reducir la tensión residual de una superficie después de la aplicación de un recubrimiento, mediante un tratamiento térmico [12].

En este trabajo se evaluaron los principales parámetros que influyen en la adherencia y en la integridad estructural de una porcelana recubierta con una aleación de cobre mediante proyección térmica oxia- 
cetilénica, obteniendo las condiciones adecuadas para mejorar la unión de la capa depositada y evitar la fractura del sustrato. El esfuerzo de adhesión del recubrimiento de cobre a la porcelana obtenido en los ensayos de este trabajo es superior al reportado por otros autores. El proceso llevado a cabo y los resultados obtenidos son presentados a continuación.

\section{METODOLOGÍA EXPERIMENTAL}

\subsection{Preparación superficial}

Con el fin de producir la rugosidad superficial que requieren los sustratos cerámicos para facilitar la adhesión de las partículas de cobre que serán depositadas sobre ellos, se utilizó papel abrasivo $\mathrm{N}^{\circ} 100$ dejando un arado en la superficie, el arado se realizó después del secado y antes de la sinterización de las probetas de porcelana. La topografía resultante de esta preparación superficial se evaluó de acuerdo con la norma ASTM D7127 utilizando un equipo Mitutoyo referencia SJ 201, obteniendo una rugosidad media $(\mathrm{Ra})$ de $9,4 \pm 3,2 \mu \mathrm{m}$.

Para evaluar la importancia de la preparación superficial, se realizaron proyecciones sobre probetas con y sin preparación superficial. En la Fig. 1 se muestra una imagen de un sustrato con y sin preparación superficial.

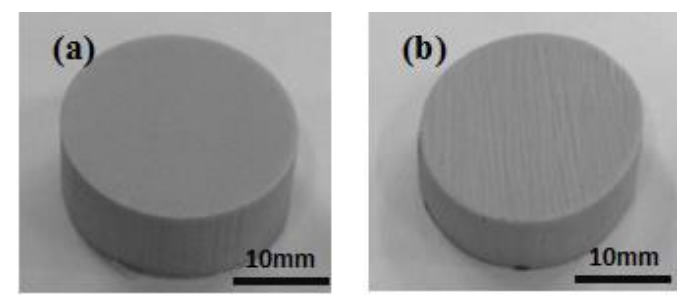

Fig. 1. Estado superficial de los sustratos cerámicos a) sin preparación superficial, b) con preparación superficial.

Fuente: Autores

\subsection{Flujo de partículas}

El flujo de las partículas de cobre fue medido con base en la norma ASTM B213, usando un flujómetro de cono (Hall
Flowmeter funnel) con una masa constante de $50 \mathrm{~g}$, la cual fluyó de forma continua durante $17,1 \pm 0,1$ segundos, dando como resultado un flujo de $2,92 \mathrm{~g} / \mathrm{s}$, el cual puede ser considerado como muy bueno.

\subsection{Evaluación de las llamas oxiacetilénicas}

Se analizaron las dos llamas (reductora y oxidante) utilizadas para elaborar los recubrimientos, con el software Jets et Poudres y los resultados obtenidos fueron verificados experimentalmente utilizando la antorcha de referencia Terodyn ${ }^{\mathrm{TM}} 2000$, la cual es fabricada por Eutectic Castolin. La llama reductora alcanzó una temperatura máxima en la zona de reacción primaria de $2979^{\circ} \mathrm{C}$ y una longitud de $1,9 \mathrm{~cm}$, mientras que la máxima temperatura en la llama oxidante fue de $2895^{\circ} \mathrm{C}$ y su longitud fue de $3,2 \mathrm{~cm}$.

\subsection{Análisis y caracterización de materias primas}

La composición química de los polvos Eutectic Castolin ${ }^{\mathrm{TM}} 21071$ utilizados para la fabricación de los recubrimientos fue determinada mediante Fluorescencia de Rayos X empleando un espectrómetro ARL OPTIMIX $^{\mathrm{TM}}$; mientras que su morfología fue analizada con un microscopio electrónico de barrido marca Jeol ${ }^{\mathrm{TM}}$ JSM-6490LV y la distribución de tamaño de las partículas que lo constituyen se determinó mediante Difracción Láser utilizando un equipo Malvern Master Sizer ${ }^{\mathrm{TM}} 2000$.

\subsection{Elaboración de recubrimientos}

Los recubrimientos fueron elaborados en la cámara de proyección térmica ARESTE I de la Universidad de Antioquia, la cual está equipada con una antorcha Eutectic Castolin Terodyn ${ }^{\mathrm{TM}} 2000$ y permite controlar las principales variables del proceso de elaboración de los recubrimientos. Las condiciones a las que se elaboraron los recubrimientos son presentadas en las Tablas 1 y 2. 
Tabla 1. Parámetros de proyección utilizando la llama carburante. Fuente: Autores

\begin{tabular}{lcc}
\hline & $\begin{array}{r}\text { Flujo de gases } \\
{[\mathrm{L} / \mathrm{min}]}\end{array}$ & $\begin{array}{c}\text { Presión de gases } \\
{[\mathrm{psi}]}\end{array}$ \\
\hline Oxígeno & 25,3 & 50 \\
Acetileno & 22 & 12 \\
Nitrógeno & 17 & 40 \\
Aire & $\mathrm{NA}$ & 30 \\
\hline Distancia de proyección [cm] & 15 \\
Flujo de polvos [g/min] & 32 \\
Pases de precalentamiento & 3 \\
Pases de proyección & 7 \\
Temperatura de precalentamiento & 148,3 \\
sustrato [ ${ }^{\circ}$ C & 0,7 \\
Velocidad de antorcha [cm/s] & 116 \\
Velocidad de portamuestras [rpm]
\end{tabular}

Tabla 2. Parámetros de proyección utilizando la llama oxidante. Fuente: Autores

\begin{tabular}{lcc}
\hline & $\begin{array}{c}\text { Flujo de gases } \\
{[\mathrm{L} / \mathrm{min}]}\end{array}$ & $\begin{array}{c}\text { Presión de gases } \\
{[\mathrm{psi}]}\end{array}$ \\
\hline Oxígeno & 55 & 50 \\
Acetileno & 22 & 12 \\
Nitrógeno & 17 & 40 \\
Aire & $\mathrm{NA}$ & 30 \\
\hline Distancia de proyección [cm] & 15 \\
Flujo de polvos [g/min] & 31,2 \\
Pases de precalentamiento & 3 \\
Pases de proyección & 3 \\
Temperatura de precalentamiento & 179 \\
sustrato [ ${ }^{\circ}$ C & 0,7 \\
Velocidad de antorcha [cm/s] & 116 \\
Velocidad de portamuestras [rpm]
\end{tabular}

\subsection{Ensayo de adherencia}

Se realizaron ensayos de adherencia a los recubrimientos obtenidos mediante el método de Pull-Off, de acuerdo con la norma ASTM D4541 (Standard Test Method for Pull-Off Strength of Coatings Using Portable Adhesion Testers).

Es de indicar que la adherencia de los recubrimientos depositados por proyección térmica normalmente es evaluada de acuerdo con la norma ASTM C633, sin embargo, en este caso no fue posible tener en cuenta los lineamientos establecidos en ella dada la naturaleza cerámica de los sustratos utilizados en este trabajo.

\section{RESULTADOS Y DISCUSIÓN}

\subsection{Caracterización de materias primas}

De acuerdo con los resultados del análisis químico se pudo establecer que los polvos Eutectic Castolin ${ }^{\mathrm{TM}} 21071$ están constituidos mayoritariamente por cobre y aluminio. Ver Tabla 3.

Tabla 3. Resultados análisis de fluorescencia de rayos X. Fuente: Autores.

\begin{tabular}{cc}
\hline \multicolumn{2}{c}{ Polvos EC ${ }^{\mathrm{TM}} 21071$} \\
\hline Elemento & Porcentaje en peso \\
\hline $\mathrm{Cu}$ & $88,66 \pm 0,16$ \\
$\mathrm{Al}$ & $8,81 \pm 0,14$ \\
$\mathrm{Fe}$ & $1,68 \pm 0,06$ \\
$\mathrm{Ni}$ & $0,155 \pm 0,008$ \\
$\mathrm{Cr}$ & $0,139 \pm 0,007$ \\
$\mathrm{Zn}$ & $0,128 \pm 0,009$ \\
$\mathrm{Mg}$ & $0,111 \pm 0,026$ \\
\hline
\end{tabular}

Los otros elementos identificados en las materias primas se encuentran en bajas cantidades (menor al 1,8\% en peso), algunos de ellos pueden ser considerados como impurezas y otros como elementos de aleación que podrían contribuir al mejoramiento de la resistencia mecánica, así como a la resistencia a la corrosión, y a soportar los cambios de temperatura del recubrimiento.

En la Fig. 2 se presenta la morfología de los polvos Eutectic Castolin ${ }^{\mathrm{TM}}$ 21071, en la que se puede apreciar que las partículas tienen forma esférica, lo cual favorece su fluidez en los conductos de alimentación a la antorcha de proyección térmica y que además explica el porqué del excelente flujo medido según la norma ASTM B213, cuyos resultados fueron presentados anteriormente.

Los resultados del análisis de distribución del tamaño de las partículas del polvo Eutectic Castolin $^{\mathrm{TM}} 21071$ indican que su $\mathrm{d}_{10}=60,1 \mu \mathrm{m}$ y el $\mathrm{d}_{90}=135,9 \mu \mathrm{m}$, siendo el tamaño de la mayor cantidad de partículas cercano a $90,2 \mu \mathrm{m}$. 


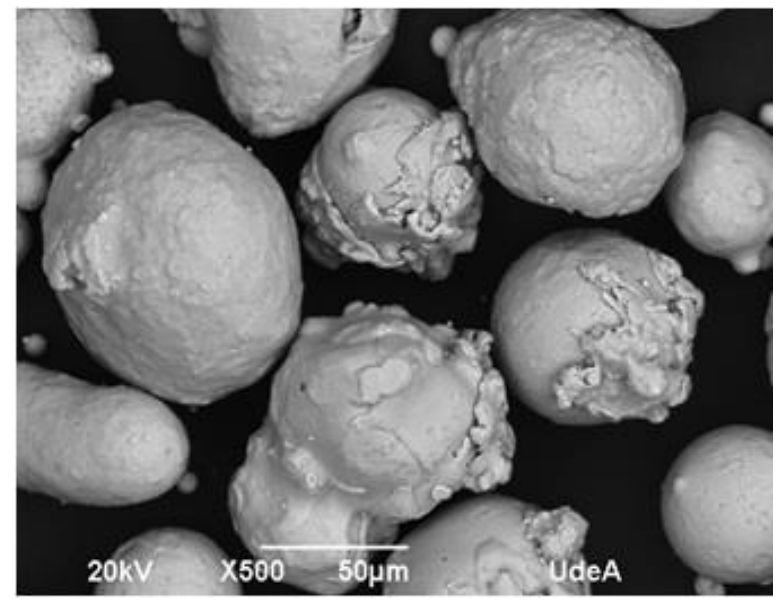

Fig. 2. Imagen SEM de la morfología de las partículas del polvo Eutectic Castolin ${ }^{\mathrm{TM}}$ 21071. Fuente: Autores

La distribución del tamaño de las partículas influye en su fluidez dentro de los conductos que las transportan hacia la antorcha y a su vez tiene un efecto significativo en la estructura del recubrimiento obtenido.

Esta distribución es muy estrecha, por lo que se puede ver favorecido el flujo de los polvos; y el tamaño de los mismos es adecuado para asegurar la continuidad del recubrimiento y la total fusión de las partículas, ya que partículas demasiado pequeñas (normalmente inferiores a $5 \mu \mathrm{m}$ ) dificultan su transporte por los conductos de la antorcha de proyección, dando como resultado recubrimientos discontinuos, mientras que partículas demasiado grandes dificultan su fusión en la llama, dando como resultado recubrimientos con partículas parcialmente fundida $y$ demasiada porosidad [7].

\subsection{Recubrimientos obtenidos}

En la Fig. 3 se muestra la estructura representativa de los recubrimientos obtenidos con las dos relaciones de gases utilizadas. En la Fig. 3 se puede apreciar la buena interacción del recubrimiento con el sustrato lo que se evidencia en la capilaridad del mismo que permitió mojar cada valle y pico de la superficie del sustrato cerámico.

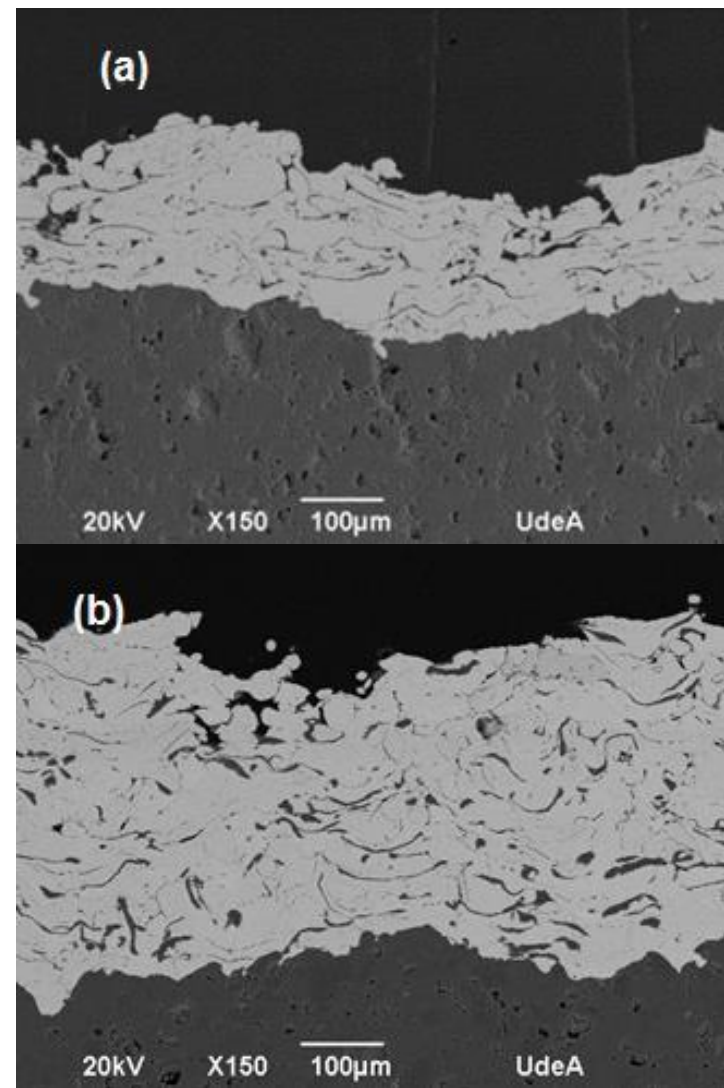

Fig. 3. Imagen SEM de la estructura representativa de la sección transversal de los recubrimientos elaborados. a) Utilizando la llama carburante, (b) Utilizando la llama oxidante. Fuente: Autores

Adicionalmente, se observan óxidos, que son característicos en este tipo de recubrimientos, debido a la interacción a alta temperatura del metal con el oxígeno del medio en el que se realizó el proceso de proyección térmica. Así mismo, es evidente la formación y definición de las lamelles, producto de una mayor fusión de las partículas utilizando la llama oxidante.

El espesor obtenido para la capa depositada a partir de la llama carburante fue de $255,9 \pm 48,7 \mu \mathrm{m}$, mientras que el del recubrimiento elaborado con la llama oxidante fue de $175 \pm 50 \mu \mathrm{m}$. El mayor espesor obtenido en la capa depositada con llama carburante se debe a que este recubrimiento se depositó en 7 pases de la antorcha frente al sustrato, mientras que para el elaborado con la llama oxidante tan solo se utilizaron 3 pases, ver Tablas 1 y 2 . Los dos espesores obtenidos pueden ser considerados como 
adecuados para asegurar la continuidad y conductividad del recubrimiento sin comprometer las propiedades mecánicas ni la estructura del sustrato [3], [4], [6].

\subsection{Resultados de adherencia}

Los resultados del ensayo de adherencia realizado mediante Pull-Off a los recubrimientos depositados sobre sustratos preparados superficialmente indican que aquellos elaborados con la llama carburante (relación de acetileno:oxígeno de 1:1,15) resisten un esfuerzo de 1,8 $\pm 0,3 \mathrm{MPa}$ antes de desprenderse del sustrato cerámico, mientras que los elaborados con la llama oxidante (relación de acetileno:oxígeno de $1: 2,5)$ soportan $2,9 \pm 0,9 \mathrm{MPa}$. Por su parte, los recubrimientos depositados sobre sustratos sin preparación superficial se desprendieron, en su mayoría, antes de realizar la prueba de Pull-Off. En todos los casos las fallas en los sistemas de recubrimientos fueron adhesivas, lo que da cuenta de una buena homogeneidad estructural del recubrimiento y por lo tanto una buena resistencia cohesiva.

A pesar de los bajos valores de adhesión obtenidos, se puede considerar que el procedimiento utilizado para elaborar los recubrimientos (especialmente utilizando una llama oxidante) base cobre sobre sustratos cerámicos de porcelana es adecuado, dadas las dificultades que se presentan para la unión de un material cerámico con un metal a causa de las diferencias en sus coeficientes de dilatación térmica y de sus temperaturas [9], [11], [12], lo cual es más crítico si quien experimenta la mayor temperatura es el metal, como en este caso en el que las partículas de cobre se fundieron, mientras que la superficie del cerámico no superó los $200^{\circ} \mathrm{C}$ (ver Tablas 1 y 2). Otros investigadores han obtenido valores de adherencia en recubrimientos de cobre depositados sobre sustratos cerámicos de tan sólo 1,7 MPa [13].

Adicionalmente, los valores de adhesión alcanzados para los recubrimientos estu- diados pueden estar afectados por el tipo de ensayo, ya que la prueba de Pull-Off realizada de acuerdo con la norma ASTM D4541 no es la más adecuada para evaluar recubrimientos de este tipo dado que la velocidad a la cual es aplicada la carga durante el ensayo es muy alta y no es controlada, como lo especifica la norma ASTM C633 propuesta para recubrimientos elaborados por proyección térmica.

\section{CONCLUSIONES}

La preparación superficial de los sustratos es de vital importancia en la elaboración de recubrimientos mediante proyección térmica, aún en el caso de los sustratos cerámicos; ya que ella brinda la rugosidad necesaria para el anclaje de las partículas al sustrato, lo cual se vio reflejado en los valores de adherencia obtenidos.

Los recubrimientos depositados sobre los sustratos a los que no se les realizó una preparación superficial previa, se desprendieron en su mayoría inmediatamente después de la proyección, evidenciando así la necesidad de una buena preparación superficial. De las relaciones de gases utilizadas, con la que se obtuvo mejores resultados tanto estructurales, como de adhesión, fue la oxidante con una relación volumétrica de oxígeno/acetileno de 2,5.

La zona de reacción primaria de la llama reductora es más corta que la correspondiente a la llama oxidante y a pesar de que la temperatura máxima alcanzada en la llama reductora fue ligeramente superior a la de la oxidante, las partículas de la aleación de cobre se fundieron en menor proporción que en la llama oxidante, debido a que el tiempo de residencia de las partículas en la zona más caliente es menor en la llama reductora dado que su longitud es más corta. Lo anterior explica la menor homogeneidad estructural y la más baja adherencia en los recubrimientos elaborados con la llama reductora. 


\section{AGRADECIMIENTOS}

Los autores agradecen al CODI por el apoyo económico brindado a través del proyecto PR14-2-07 y a la empresa Electroporcelana Gamma que junto con la Universidad de Antioquia financiaron el proyecto PI14-1-01.

\section{REFERENCIAS}

[1] E. Kuffel, R. N. Allan, and I. Aktekin, "Radio interference from high-voltage insulators and factors which affect the interference level," Proc. Inst. Electr. Eng., vol. 112, no. 5, p. 925, 1965.

[2] R. C. McCune, "Potential Applications of ColdSpray Technology in Automotive Manufacturing," in Thermal Spray: Advancing the Science and Applying the Technology, 2003.

[3] N. Jordan, R. Schröder, H. Harig, and R. Kienzler, "Influences of the spray deposition process on the properties of copper and copper alloys," Mater. Sci. Eng. A, vol. 326, no. 1, pp. 51-62, Mar. 2002.

[4] P. L. Fauchais, J. V. R. Heberlein, and M. I. Boulos, Thermal Spray Fundamentals. Springer US, 2014.
[5] ASME, Surface texture (surface roughness, waviness, and lay). New York, N.Y.: The Society, 1986, p. 43.

[6] L. Pawlowski, The Science and Engineering of Thermal Spray Coatings. John Wiley and Sons, 2008.

[7] J. R. Davis, Handbook of Thermal Spray Technology. ASM International, 2004.

[8] F. Vargas, "Elaboration de couches céramiques épaisses à structures micrométriques et nanométriques par projections thermiques pour des applications tribologiques," Université de Limoges, 2010.

[9] F. Ernst, "Metal-oxide interfaces," Materials Science and Engineering: R: Reports, vol. 14, no. 3. pp. 97-156, Apr-1995.

[10]M. Rühle, "Structure and composition of metal/ceramic interfaces," J. Eur. Ceram. Soc., vol. 16, no. 3, pp. 353-365, Jan. 1996.

[11] J. E. Kuneman and J. F. Dickson, "Direct bond copper ceramic substrate for electronic applications," US4563383 A, 1984.

[12] M. Kinsho, Y. Saito, Z. Kabeya, K. Tajiri, T. Nakamura, K. Abe, T. Nagayama, D. Nishizawa, and N. Ogiwara, "Development of alumina ceramics vacuum duct for the $3 \mathrm{GeV}$-RCS of the J-PARC project," in Vacuum, 2004, vol. 73, pp. 187-193.

[13] K. F. Zsamboky, "Metallized ceramic substrate and method therefor," US Patent 5058799 A, 1991. 